\title{
CrimRxiv
}

\section{Assessing and Responding to the Recent Homicide Rise in the United States}

Richard Rosenfeld, Shytierra Gaston, Howard Spivak, Seri Irazola

Published on: Jul 07, 2021

License: Creative Commons Attribution 4.0 International License (CC-BY 4.0). 
